\title{
Input power-mechanism relationship for ultrasonic irradiation: Food and polymer applications
}

\author{
Mohammad Reza Kasaai
}

Department of Food Science and Technology, Faculty of Agricultural Engineering, Sari Agricultural Sciences and Natural Resources University, Sari, Iran; reza kasaai@hotmail.com

Received 8 June 2013; revised 8 July 2013; accepted 15 July 2013

Copyright (C) 2013 Mohammad Reza Kasaai. This is an open access article distributed under the Creative Commons Attribution License, which permits unrestricted use, distribution, and reproduction in any medium, provided the original work is properly cited.

\begin{abstract}
Mechanisms for interactions between ultrasound waves and materials vary as a function of input power of ultrasound. The objectives of this study were to compare mode of actions for ultrasound waves at different input powers. This study also describes various effects of ultrasound on materials at different input powers with emphasize on food and polymer applications. At low power of ultrasound, the major mechanism is acoustic streaming and at a power above threshold value, the most possible one is acoustic cavitation. Low power of ultrasound is a powerful analytical technique to investigate on physico-chemical properties of both biological and non-biological materials. While at sufficiently high power, it generates shear forces that are able to create different effects. For each pair of medium-acoustic wave, two types of mechanisms, acoustic streaming and cavitation may be occurred simultaneously.
\end{abstract}

Keywords: Ultrasound; Energy; Mechanism; Acoustic Streaming; Cavitation

\section{INTRODUCTION}

Sound waves with frequencies higher than those can be heard by human $(>16 \mathrm{kHz})$ is called ultrasound. The upper limit of ultrasonic frequency is not sharply defined. It is usually taken to be $5 \mathrm{MHz}$ for gases and $500 \mathrm{MHz}$ for liquids and solids [1]. $20 \mathrm{kHz}$ is usually taken as the lower frequency limit of ultrasound waves [2]. Ultrasound waves may be divided into two main areas [1,3]: 1) low amplitude (high frequency/low power) propagation is concerned with the effect of medium on the wave, low amplitude waves are used to measure the velocity and absorption coefficient of the wave in the medium; and 2) high energy (low frequency) propagation is concerned with the effect of the wave on medium.

Low intensity (intensity is actual output power per surface area, $\mathrm{W} \cdot \mathrm{cm}^{-2}$ ) is a powerful analytical technique for investigating the physico-chemical properties of many biological and non-biological materials [4]. Ultrasonic irradiation at low power did not induce any chemical changes, but at a power above a threshold value, chemical changes have been created $[5,6]$. High power ultrasound induces a permanent chemical or physical change on a material [3]. High energy input produces cavitation and micro-streaming in liquids, heating, and surface-instability effects at liquid-liquid and liquid-gas interfaces $[3,7,8]$.

Use of sonication energy provides a better way to reach higher efficiency, higher rates for chemical, physical, or physico-chemical process, shorter processing times and change reaction pathways. Knowledge on mechanism of ultrasound provides a way to investigate some of fundamental properties of materials. An understanding of mechanisms for different (chemical, physical, biological) effects of sound, is important in connection with its applications in different fields (medicine, food, chemistry). Knowledge on mechanisms of ultrasound is essential because of practical applications. There is considerable need to increase understanding of mechanisms in order to evaluate performances and limitations involved in its various applications.

This study describes mode of actions for ultrasound versus input energy. This work also provides different views and explanations on the mechanisms of ultrasound irradiation as a function of input energy with particular attention in the field of polymer degradation and food applications.

\section{THEOREORETICAL CONSIDERATION}

Conversion of Electrical Energy into Physical, Chemical or Biological Effects

The ultrasonic system transforms electrical power into 
vibrational energy, i.e. mechanical energy [9]. The mechanical energy is then transmitted into a sonicated medium. A part of input energy is lost through conversion into heat, and another part produces cavitation. A fraction of cavitational energy produces chemical, physical, or biological effects. Other fractions are reflected and consumed in sound re-emission (harmonics and sub-harmonics). The conversion of electrical energy into ultrasonic effects was illustrated in Figure 1. The effects as a measure of ultrasonic yield can be defined as follows [9]:

$$
\text { Ultrasonic yield }=\text { measured effect/input power }
$$

To estimate the output power, the conversion yield of the ultrasonic system has to be known. It is not easy to determine output power, since part of energy is reflected back to the transducer. In a pure liquid (specific case), it might be assumed that almost all of the mechanical energy produces heat and so, via calorimetry output power can be obtained [9]. Increasing the power increases the reaction yield [10]. The ultrasonic yield increases with an increase in ultrasonic power. The yield is dramatically increased near the caviation power threshold and then remains nearly constant [10].

Practically, ultrasonic propagation is mostly non-linear phenomenon to some extent. The degree of non-linearity is related to several factors including ultrasonic parameters such as ultrasonic intensity, I, (the energy transmitted per unit time per unit normal area of fluid). The intensity of wave is proportional to square of the maximum pressure amplitude of wave $\left(\mathrm{P}_{\mathrm{A}}\right)$ and is described in Equation (2).

$$
\mathrm{I}=\mathrm{P}_{\mathrm{A}}^{2}(2 \rho \cdot \mathrm{c})^{-1}
$$

where $\rho$ is density of a medium and $\mathrm{c}$ is velocity of sound in that medium [11]. The acoustic pressure is a sinusoidal wave dependent on time $(t)$, frequency $(f)$ and

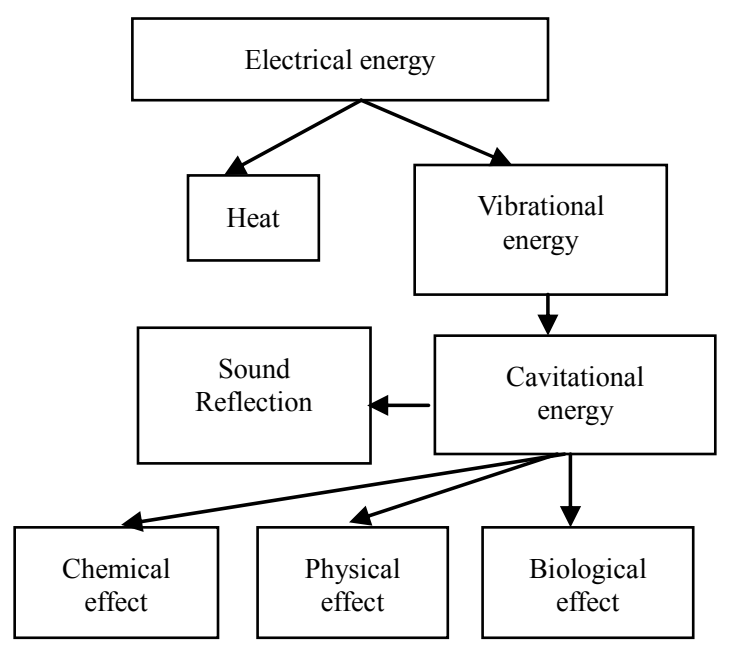

Figure 1. Conversion of electrical energy into physical, chemical or biological effects. the maximum pressure amplitude of the wave, $\mathrm{P}_{\mathrm{a}, \max }$ and is represented by the following equation $[12,13]$ :

$$
\mathrm{P}_{\mathrm{a}}=\mathrm{P}_{\mathrm{a}, \max } \sin (2 \pi \mathrm{ft})
$$

The maximum pressure amplitude of the wave $\left(\mathrm{P}_{\mathrm{a}, \max }\right)$ is directly proportional to the input power of the transducer. A sufficiently large increase in the intensity of ultrasound will generate larger values of acoustic pressure. Increase in the value of $\mathrm{P}_{\mathrm{a}}$ lead to a more and violent collapse [11] and consequently larger values for chemical or physical effects. A large value of intensity may also generate shock wave and consequently results in drastic effect on the chemical or physical effects [14]. At low intensity (amplitude), the pressure wave induces motion and mixing within the fluid, so called acoustic streaming [15]. At higher intensities, the local pressure in the expansion phase of the cycle falls below the vapour pressure of the liquid, causing tiny bubbles to grow (created from existing gas nuclei within the fluid). A further increase generates negative transient pressures within the fluid, enhancing bubble growth and producing new cavities by the tensioning effect on the fluid [16].

The ultrasonic velocity of material, $\mathrm{C}$, is related to its physical properties by the following equation:

$$
\mathrm{C}^{2}=\mathrm{E} / \mathrm{d}
$$

where $\mathrm{E}$ and $\mathrm{d}$ are elastic modulus and density, respectively [4]. Two most commonly measured ultrasonic parameters at low power are ultrasonic velocity (C) and attenuation coefficient $(\alpha)$. The two parameters are related to the physico-chemical properties of the material through which they propagate and can therefore be used to provide information about these properties [4]. The greatest amount of information can be obtained by measuring both $\mathrm{C}$ and $\alpha$. In many applications, however, it is only necessary to measure either $\mathrm{C}$ or $\alpha$, e.g. the degree of crystallization of a fat can be determined by measuring the velocity at a single frequency [4].

\section{MECHANISM OF ULTRASONIC IRRADIATION}

A created micro-bubble contains vapor from a solvent or any volatile reagent. On collapse, these vapors are subjected to the enormous increases in both temperature and pressure. The solvent and/or the reagent generate reactive species of the radicals. Shock wave produces by bubble collapse, or even by propagating ultrasonic wave itself, could act to disrupt solvent structure and could influence reactivity by altering solvation of the reactive species present [17].

Different views and explanations on mechanisms for ultrasonic degradation of polymers have been already proposed as follows: 1) macro-molecule radicals have been observed for many carbon skeleton polymers as 
discussed by Henglein [18,19]; 2) cleavage of a covalent bond can occur in two ways, homolytically resulting in one electron from the bond going to each fragment to produce radical species, or heterotytically, with both electrons going to one fragment, leading to formation of an ion pair. Both of these possibilities have been observed during polymer degradation [20]; 3) chain breakage was due to inertial or frictional forces between the solvent and the polymer since they transmit to ultrasound differently [20]; 4) in an aqueous polymer solution, water is partially dissociates into $\mathrm{OH}$ radicals. Some of these radicals diffuse out of the cavity to the surrounding liquid, where they can react with solute molecules. This mechanism makes sono-chemistry similar, in a sense, to radiation chemistry, where at first solvent radicals are generated, subsequently the latter attack to the solute [21]. Polymers in solution may undergo pyrolysis in the hot interfacial region between the bubble and the surrounding liquid; 5) ultrasonic degradation is based on cavitation effects produced by ultrasound waves; 6) degradation as well as depolymerisation occurs as a result of elongational flow fields, thus producing a less viscous polymer [22]; 7) when a polymer solution is exposed to ultrasound, micro-bubbles containing dissolved gas or vapor of volatile liquids are created and the bubbles grow and finally collapse in a short time as short as microsecond [23-25]; 8) radicals were detected by spin-trapping method and dosimeters when a polymer solution was exposed to ultrasonic irradiation [26,27]; 9) a shear deformation during collapse of the bubbles in the vicinity of cavitation seems to be responsible for disintegration of polymers in organic solvents [5]; 10) Kanwal et al., [28] noted that both cavitation and viscoelastic response of a polymer molecule in solution are responsible for ultrasonic degradation; 11) the degradation is mechanical in nature, although secondary chemical reactions, for example, radical species may be important in some cases. A combination of solvent flows and shock waves around cavitation bubbles serves to initially stretch out the polymer chains into extended conformations and finally to generate sufficient stress to break a bond in the polymer backbone [20]; 12) the mechanism of ultrasonic degradation of a polysaccharide $(20 \mathrm{kHz}, 100-1500 \mathrm{~W})$ has been explained by more with mechanical and less with radical effects [29]. The degradation mechanism has been attributed to cavitation (mechanical effect); that is, the formation and collapse of microscopic vapor bubbles generated by strong sound waves [30]. Lorimer \& Mason [1] in their review article concluded that the experimental evidences suggest that degradation is caused by: 1) the hydrodynamic forces of cavitation (i.e., the shock wave energies produced on bubble implosion); 2) the shear stresses at the interface of pulsating bubbles; or 3) the associated thermal and chemical effects of both stable and transient cavitation, since all of the above are dependent upon the same factors, i.e. intensity, frequency, gas content and type.

Lorimer and Mason [1] concluded that increase in frequency of the ultrasound leads to a decrease in the extent of depolymerization. Two frequencies $(20 \mathrm{kHz}$, and $1.7 \mathrm{MHz}$ ) have been used to produce ultrasonic fields. At the lower frequency, cavitation effects dominate. At the higher frequency, free radical formation drives the reactions. Applying these effects simultaneously combines physical and chemical forces.

The ultrasonic degradation has several aspects that differentiate it from thermal or photochemical processes and that may be regarded as characteristic of the effect of ultrasound. It proceeds faster at high molecular weights and shows down until, at some limiting value, degradation ceases. In addition, ultrasonic degradation is the cleavage of the polymer chain near the center of the molecule. This property means that ultrasound can be used for controlled degradation to give polymers with specific properties [20]. Ultrasonic power was a major factor in fragmentation of macromolecules among several ultrasonic parameters. Chain scission increased with an increase in power of ultrasound.

\subsection{Cavitation}

When a liquid is subjected to the action of ultrasound, a rupture in the liquid occurs in a form of a small bubble filled with vapor (and gas, if present in the liquid as a solute, as well as molecules of any other volatile solutes). The formation of these bubbles and their subsequent collapse is called cavitation [31]. Among non-thermal mechanisms, the most possible one in many aspects is acoustic cavitation: Ultrasound with sufficient energy produces cavitiation, starting with nucleation followed by growth and collapse of microscopic bubbles [32-34]. Ultrasonic power induces cavitation in a medium and the collapse of cavitation bubbles produces very high local temperatures and pressures [9].

The exact quantification of cavitation phenomena is complex, but the acoustic pressure necessary to produce bubbles depends primarily on the hydrostatic pressure over the liquid and on the surface tension of the liquid [20]. Cavitation bubbles actually form at acoustic pressures considerably lower than those needed to break the tensile strength of a liquid [20]. If the rarefaction cycle of the acoustic wave leads to a sufficiently large negative pressure, then the intermolecular forces holding the molecules together can be overcome, leading to the formation of a bubble [20]. A power density from less than $1 \mathrm{~W}$ is required to produce cavitation [3]. Cavitation is almost suppressed at frequencies about $2 \mathrm{MHz}$. It has been attributed to the bubbles not having sufficient time to grow and collapse between successive waves at very 
high frequencies.

There are two forms of cavitation-transient and stable. The two types of cavitation are described in the following:

\subsubsection{Transient Cavitation}

Transient cavitation bubbles are voids, or bubbles filled with vapor [1]. It is produced using ultrasonic intensities in excess of $10 \mathrm{~W} \cdot \mathrm{cm}^{-2}$ They exist one or a few acoustic cycles, expanding to a radius of at least twice their initial sizes before collapsing violently on compression and often disintegrating into smaller bubbles [1]. The smaller bubbles may act as nuclei for further bubbles, or for sufficiently small radius, $\mathrm{R}$, they can dissolve into a solution because of very large pressure due to surface tension. Transient cavities generally exist for less than a single acoustic cycle during which they expand to at least double their initial size before collapsing violently into smaller bubbles [1]. Different effects such as erosion, emulsification, molecular degradation, sono-luminescence, and sono-chemical enhancement of reactivity are entirely attributed to the collapse of transient cavities. It is generally assumed that the transient bubbles are mainly responsible for sono-chemical effects [3].

\subsubsection{Stable Cavitation}

Stable cavities or stable bubbles are those which oscillate (often non-linearly) many times with only small changes in diameter about the equilibrium size $[1,3]$. The bubbles ultimately grow in size after too many times oscillation and finally collapse [3]. Stable bubbles contain mainly gas and some vapor and are produced at fairly low intensities $\left(1-3 \mathrm{~W} \cdot \mathrm{cm}^{-2}\right)$. The time scale over which they exist is sufficiently long that mass diffusion of gas, as well as thermal diffusion, with consequent evaporation and condensation of the vapor, can occur, resulting in significant long-term effects. If the rates of mass transfer, across the gas-liquid interface, are not equal, it may result in bubble growth. The mechanism by which small micro-bubbles in the liquid grows is termed rectified diffusion. In the expansion phase of the acoustic cycle gas diffuses from the liquid into the bubble, whilst in the compression phase, gas diffuses out of the bubble into the liquid. Since the interface area is greater in the expanded phase, the inward diffusion is greater, leading to an overall growth of the bubble. As the bubble grows the acoustical and environmental conditions of the medium will change, the medium becomes acoustically more compressible [1]. The stable bubble may be transformed into a transient bubble and undergo collapse. The violence of collapse, however, will be less than that of transient bubbles filled by vapors, since the gas will cushion the implosion. The cavity will reduce to a minimum size, $R_{\min }$, during compression, after which it will expand to $\mathrm{R}_{\max }$ and subsequently oscillate between these extremes [1]. On the other hand, the bubbles may continue to grow during subsequent cycles until they are sufficiently buoyant to float to the surface and be expelled (this is the process of ultrasonic degassing) [1]. Stable bubbles are also capable of being transformed into cavities [1].

\subsection{Effect of Frequency on Mode of Action}

Ultrasound (i.e., mechanical waves at a frequency above the threshold of human hearing) can be divided into three frequency ranges: 1) low frequency (usually high intensity or high power) (16 - $100 \mathrm{kHz}) ; 2)$ high frequency (usually low intensity or low power) $(100 \mathrm{kHz}$ - $2 \mathrm{MHz}$ ); and 3) diagnostic ultrasound (2 - $20 \mathrm{MHz}$ ). The frequency is inversely proportional to the bubble sizes $[1,8,35]$. Low frequency ultrasound $(16-100 \mathrm{kHz})$ generates large cavitation bubbles and results in high temperatures and pressures in the cavitation zone. Low frequency ultrasound has remarkable effects on many chemical reactions and physical changes. To achieve these effects, a relatively high power density (from 1 $\mathrm{W} \cdot \mathrm{cm}^{-2}$ to thousands of $\mathrm{W} \cdot \mathrm{cm}^{-2}$ ) is required. This input energy produces cavitation and micro-streaming in liquids, heating and fatigue in solids, and surface-instability effects at liquid-liquid and liquid-gas interfaces $[3,7]$.

As the frequency increases the cavitation zone becomes less violent and in the $\mathrm{MHz}$ range no cavitation is observed and the main mechanism is acoustic streaming [13]. The creation of cavitation in liquids decreases with an increase in frequency. At very high frequency, where the rarefaction (and compression) cycles are very short, the finite time required for the rarefaction cycle is too small to permit a bubble to grow to a size sufficient to cause disruption of the liquid [1]. Most industrial applications (a wide variety of chemical or food processes as well as cleaning) operate between 16 and $100 \mathrm{kHz}$, because cavitation can be produced within this frequency range.

\section{DISCUSSION}

\subsection{Chemical Effects}

Sono-chemical reactions in liquids arise from the process, starting with nucleation followed by growth and collapse of microscopic bubbles, called cavitation [32, 34]. One of major consequence of cavitation is formation of radicals from decomposition of solvents or other dissolved species [24]. The major effect of sonication of water, above the cavitation threshold, is hemolytic bond cleavage to form hydrogen, $\mathrm{H}^{*}$, and hydroxyl, $\mathrm{OH}^{*}$ radicals. There is both indirect evidence for these radicals, such as the formation of hydrogen peroxide, and direct evidence, from the electron spin resonance spectra of 
trapped species. These radicals are very reactive and readily react with other dissolved species, making the process potentially very useful. Hence, monitoring and quantifying the radials formed is important in understanding mechanism and applicability of such processes $[27,36,37]$.

The effect of ultrasonic power $(20 \mathrm{kHz}, 100-1500 \mathrm{~W})$ on degradation of polysaccharide from red algae in the experimental time range (from 0 to $240 \mathrm{~min}$ ) was: the intrinsic viscosity decreased with an increase in ultrasonic power [29]. The authors concluded that the effect of power on the sonolysis of the polysaccharide may be explained mainly by mechanical and less by radical effects [29]. However, when the polysaccharide was degraded at a fixed ultrasonic power and frequency to a certain molecular weight, mechanical degradation cannot continue. Similar results can be found in ultrasonic degradation of dextrans, sodium dodecylbenzene sulfonate, and azo dye [38-40]. Thus, at low frequency (for example. $20 \mathrm{kHz}$ ), major effect is mainly mechanical, and at higher frequencies $(>500 \mathrm{kHz})$ the major effect for degradation are radical reactions [41]. Ultrasonic degradation of starch was performed under conditions $(20 \mathrm{kHz}$, out power was $400 \mathrm{~W}$, density of ultrasonic output was, $80 \mathrm{~W} \cdot \mathrm{cm}^{-2}$ ) [22]. Hydrolytic degradation of chitosan was accelerated by ultrasound $\left(35 \mathrm{kHz}, 2 \mathrm{~W} \cdot \mathrm{cm}^{-2}\right)$ [42]. Degradation of alginate and chitosan solutions by ultrasonic irradiation has been studied [43]. The authors reported that the molecules become less mobile in the concentrated solution, therefore, it is more difficult for cavitation bubbles to form. The velocity gradients around the collapsing bubbles as well as the stress generated in the polymer chains become smaller $[44,45]$.

Ultrasonic irradiation has been used to reduce molecular weight of wheat starch, as determined by changes in its gel filtration profiles [46]. Degradation of waxy rice starch, a highly branched polymer, was observed upon ultrasonic irradiation. The rate of degradation was accelerated at or above the gelatinisation temperature of starch [47]. Effects of ultrasonic irradiation $(360 \mathrm{kHz}$; power between $0-100 \mathrm{~W}$ ) on aqueous solutions of starch were studied [31]. This treatment is an efficient procedure for reduction of molecular weight of starch. This study has been shown that the yield of degradation depends on ultrasound power. It has been demonstrated that at applied ultrasound frequency degradation is caused both by $\mathrm{OH}$ radicals and mechano-chemical effects. [31]. Ultrasonic degradation is based on cavitation effects produced by ultrasound waves. Degradation as well as depolymerisation occurs as a result of elongational flow fields, thus producing a less viscous polymer [22]. The rate of degradation increased with an increase in ultrasonic power [48-50].

Ultrasound has been used to perform polymerization or depolymerization process. Low frequency of ultrasound waves $(18 \mathrm{kHz}-1 \mathrm{MHz})$ induced polymerization and depolymerization. Ultrasound with a frequency of 20 $\mathrm{kHz}$ caused both polymerizaton and depolymerization [51]. Change from depolymerization to polymerization was due to change in the nature of cavitation. Ultrasonic irradiation at low power did not induce any chemical changes, but at a power above a threshold value, chemical changes have been created $[5,6]$.

When a polymer solution is exposed to ultrasound, micro-bubbles containing dissolved gas or vapor of volatile liquids are created and the bubbles grow and finally collapse in a short time as short as a few microseconds [23-25]. Radicals were detected by spin-trapping method when a polymer solution was exposed to ultrasonic irradiation [26]. A shear deformation during collapse of the bubbles in the vicinity of cavitation seems to be responsible for disintegration of polymers in organic solvents [5]. Kanwal et al. [28] noted that both cavitation and viscoelastic response of a polymer molecule in a solution are responsible for ultrasonic degradation.

The effects of ultrasonic power on degradation of hydroxyethyl cellulose and poly (ethylene oxide) in aqueous solutions $[49,50,52]$ were investigated. They reported that the efficiency and rate of degradation process increased with an increase in ultrasonic power. Ultrasonic irradiation induced either increase or decrease a polymer solution viscosity. However, if enough energy is applied, the molecular weight is decreased causing a permanent viscosity reduction [53].

A focused ultrasound $(750 \mathrm{kHz})$ has been used for a chemical reaction (oxidation of iodide). The rate and efficiency for oxidation of the iodide by focused ultrasound was ten times higher than conventional (continuous wave) sonication. In a pulsed ultrasound field, bubbles may collapse immediately after the power is off, while in the focused ultrasound (a phased array transducer with multiple sectors and a geometric focus is able to produce focal ultrasound fields with spiral-shaped wavefronts) bubbles may collapse immediately after the direction of the spiral is changed [54]. In the pulsed ultrasound field, bubble growth does not take place during off time, while the switched focal field could perform continuous bubble recycling (growth and collapse). Focused ultrasound using periodic switching between clockwise and counter-clockwise spiral focal fields, is effective and does not require any mechanical agitation (such as rotation of a reaction vessel) for inducing sonochemical reactions.

\subsection{Physical Effects}

The effect of ultrasonic power (cuphorn system) on a heterogeneous (solid-liquid transfer) reaction has been studied [10]. The reaction yield dramatically increased 
near the cavitation power threshold $(10-12 \mathrm{~W})$ and then remains nearly constant. Ultrasonic fragmentation (20 $\mathrm{kHz}, 2.5-100 \mathrm{~W}$ ) of agglomerate particles suspended in liquids has been studied. Disintegration of agglomerares was taken place by their interaction with cavitation bubbles formed in the liquid and collapse in the ultrasonic field [55,56]. Other reports [57] showed that the agglomerates were broken up by their interaction with collapsing cavities. Another mechanism for the disintegration described, by collisions among particles of agglomerates [32]. The authors concluded that the time lag decreases with input power, and depends on the original strength of the ceramic powder and the applied cavitation pressure. Other physical effects of ultrasonic irradiation include heating of solids and liquids and formation of fogs and emulsions [3].

\subsection{Biological Effects}

When ultrasound propagates through a biological tissue, absorption occurs, and acoustic energy is converted into heat [58]. The temperature rises depending on the nature of the sound field as well as on characteristics of the tissue. The temperature elevation is high enough to produce structural or functional changes, either beneficial or adverse. When exposure to ultrasound produces a biological change that cannot be attributed to temperature rise alone, the cause may be some form of cavitation. Ultrasound caused the instant coagulation of egg albumen, the reduction of agar and starch, and promoted rapid hydrolysis of sucrose to glucose [17]. Ultrsasonic irradiation $\left(500 \mathrm{kHz}, 10 \mathrm{~mW} \cdot \mathrm{cm}^{-2}\right)$ of a biofilm enhanced killing of bacteria by gentamicin. The structure of biofilm was not changed when a biofilm was exposed to continuous ultrasound. Low intensity ultrasound does not disrupt the biofilm or disperse the bacteria. The possible use of ultrasound is to enhance the action of antibiotics against biofilms [59]. High pressure amplitudes are required to produce destructive cavitation in vivo. Cavitation tends to occur readily when suspensions of cells are exposed to ultrasound, since cavitation nuclei are usually present in these suspensions.

\section{APPLICATIONS OF ULTRASOUND}

Ultrasound with power levels of milli-watts $(\mathrm{mW})$ or below is used industrially for measuring material properties as a non-destructive, non-invasive testing and diagnostic technique $[3,4,60]$. It is used in many fields of science, engineering, food and medicine. Characterization of materials by ultrasound has advantages over many of traditional techniques: it is capable of rapid and precise measurements. It is used to characterize fats and oils (dynamic rheology and composition of oils, oil content, droplet size of emulsions and the solid fat content of partially) [4]. Low intensity ultrasound is a powerful analytical technique for investigating physico-chemical properties of many biological and non-biological materials. High frequency $(>1 \mathrm{MHz})$ with low intensity waves are useful in providing information on relaxation phenomena such as segmental motion, conformational analysis, vibrational-translational energy inter-charge and polymer solvent interactions [23].

The use of low frequency with sufficiently high power of ultrasound in industrial processes has two main requirements; a liquid medium (even if the liquid forms only $5 \%$ of the overall medium) and a source of highenergy vibrations (the ultrasound). Low frequency with sufficiently high power of ultrasound has been employed a wide variety of industries as follows: cleaning, sterilization, floatation, drying, degassing, defoaming, filtration, homogenization, emulsification, dissolution, deaggregation of powder, biological cell disruption, extraction, crystallization, chemical processes, acceleration of chemical processes, organic synthesis, and provide information on both polymerization and partial depolymerization and preparation of moderate macromolecules from large ones [9,23,33,38,48,61-67]. Applications of ultrasonic irradiation versus input power is given in Table 1.

\section{CONCLUSION}

Mode of action for ultrasound waves changes as a function of input power. Low power of ultrasound is a non-destructive and a non-invasive method, and it is a

Table 1. Applications of ultrasonic irradiation versus input power.

\begin{tabular}{|c|c|c|}
\hline Input Power & Low energy & High energy \\
\hline $\begin{array}{l}\text { Performances/ } \\
\text { characteristics }\end{array}$ & $\begin{array}{l}\text { Non-destructive; } \\
\text { non-invasive } \\
\text { testing; diagnostic } \\
\text { technique; to } \\
\text { mesure material } \\
\text { properties. }\end{array}$ & $\begin{array}{l}\text { To perform various } \\
\text { (chemical, biochemical } \\
\text { and biochemical) } \\
\text { processes; to accelerate } \\
\text { various processes. }\end{array}$ \\
\hline $\begin{array}{l}\text { Food } \\
\text { applications }\end{array}$ & $\begin{array}{l}\text { To determine } \\
\text { physico-chemical } \\
\text { properties and } \\
\text { characterization of } \\
\text { food and } \\
\text { biological } \\
\text { components. }\end{array}$ & $\begin{array}{l}\text { Sterilization, drying, } \\
\text { defoaming, filtration, } \\
\text { homogenization, } \\
\text { emulsification, dissolution, } \\
\text { de-aggregation of powder, } \\
\text { biological cell disruption, } \\
\text { extraction, crystallization, } \\
\text { acceleration of several } \\
\text { processes. }\end{array}$ \\
\hline $\begin{array}{l}\text { Polymer } \\
\text { applications }\end{array}$ & $\begin{array}{l}\text { To provide } \\
\text { information on } \\
\text { segmental motion } \\
\text { and polymer- } \\
\text { solvent } \\
\text { interactions; to } \\
\text { determine polymer } \\
\text { conformation. }\end{array}$ & $\begin{array}{l}\text { Polymerization, } \\
\text { fragmentation, } \\
\text { depolymerization, } \\
\text { acceleration of polymer } \\
\text { synthesis and } \\
\text { depolymerization. }\end{array}$ \\
\hline
\end{tabular}


powerful analytical technique to characterize and determine physico-chemical properties of food components. It has been used to characterize food materials, to determine different types and contents of food components, and droplet size of emulsions. Ultrasonic irradiation at low power did not induce any chemical or physical changes, but at a power above a threshold value, chemical or physical changes have been created. Minimum sonication energy is required to reach the cavitation threshold. This minimum depends upon the frequency. The different effects of ultrasound as well as ultrasonic yield increase with an increase in ultrasonic power. The ultrasonic yield dramatically increased near the caviation power threshold and then remained nearly constant. Ultrasonic power was a major factor in fragmentation of macromolecules among several ultrasonic parameters. Chain scission increased with an increase in power of ultrasound. There should exist a narrow range of ultrasonic power/frequency for each medium both acoustic streaming and cavitation occurred simultaneously. There should also exist a frequency as a shock frequency for each medium, and the ultrasound effect is a maximum value.

\section{REFERENCES}

[1] Lorimer, J.P. and Mason, T. J. (1987) Sonochemistry: Part 1: The physical aspects. Chemical Society Review, 16, 239-274. doi:10.1039/cs9871600239

[2] O'Brien, W.D. (2007) Ultrasound-biophysics mechanisms. Progress in Biophysics and Molecular Biological, 93, 212-255. doi:10.1016/j.pbiomolbio.2006.07.010

[3] Bremner, D. (1990) Historical introduction to ultrasound. In: Mason, T.J., Ed., Advances in Sono-Chemistry, JAI Press LTD., London, Vol. 1, 1-37.

[4] McClement, D.J. and Povey, M.J.W. (1992) Ultrasonic analysis of edible fats and oils. Ultrasonics, 30, 383-388. doi:10.1016/0041-624X(92)90094-3

[5] Kuttruff, H. (1991) Ultrasonics: Fundamentals and applications. Elsevier Applied Science, London, 363-394.

[6] Crawford, A.F. (1955) Ultrasonic engineering with particular references to high power applications. Butterworths Scientific, London, 202-215.

[7] Shoh, A. (1983) Ultrasonics. In: Kirk-Othmer, Encyclopedia of Chemical Technology, Vol. 24, New York, Wiley, 462-479.

[8] Mason, T.J. (1990) Sonochemistry: The uses of ultrasound in chemistry. The Royal Society of Chemistry, Cambridge.

[9] Berlan, J. and Mason, T.J. (1992) Sonochemistry: From research laboratories to industrial plants. Ultrasonics, 30, 203-212. doi:10.1016/0041-624X(92)90078-Z

[10] Ratoarinoro, N., Wilhelm, A.M., Berlan, J. and Delmas, H. (1992) Effects of ultrasound emitter type and power on a heterogeneous reaction. The Chemical Engineering Jour- nal, 50, 27-31. doi:10.1016/0300-9467(92)80003-S

[11] Tsaih, M.-L., Tseng, L.Z. and Chen, R.H. (2004) Effects of removing small fragments with ultrafiltration treatment and ultrasonic conditions on the degradation kinetics of chitosan. Polymer Degradation and Stability, 86, 25-32. doi:10.1016/j.polymdegradstab.2003.10.015

[12] Muthukumaran, S., Kentish, S.E., Stevens, G.W. and Ashokkumar, M. (2006) Application of ultrasound in membrane separation processes: A review. Reviews in Chemical Engineering, 22, 155-194. doi:10.1515/REVCE.2006.22.3.155

[13] Patist, A. and Bates, D. (2008) Ultrasonic innovations in the food industry: From the laboratory to commercial production. Innovative Food Science and Emerging Technologies, 9, 147-154. doi:10.1016/j.ifset.2007.07.004

[14] Baxter, S., Zivanovic, S. and Weiss, J. (2005) Molecular weight and degree of acetylation of high-intensity ultrasonicated chitosan. Food Hydroclloids, 19, 821-830. doi:10.1016/j.foodhyd.2004.11.002

[15] Leighton, T.G. (1995) Bubble population phenomena in acoustic cavitation. Ultrasonics Sonochemistry, 2, S123S136. doi:10.1016/1350-4177(95)00021-W

[16] Mason, T.J. (1998) Power ultrasound in food processingThe way forward. In: Povey, M.J.W. and Mason, T.J., Eds., Ultrasound in Food Processing, Blackie Academic \& Professional, London, 103-126.

[17] Lindley, J. and Mason, T.J. (1987) Sonochemistry: Part 2: synthetic applications. Chemical Society Review, 16, 275311. doi: $10.1039 / \operatorname{cs} 9871600275$

[18] Henglein, V.A. (1954) Die auslösung und der verlauf der polymerisation des acrylamids unter dem einfluß von ultraschallwellen. Die Makromolekulare Chemie, 14, 15-39. doi:10.1002/macp.1954.020140102

[19] Henglein. V.A. (1955) Die reaktion des $\alpha, \alpha$-diphenyl- $\beta$ pikryl-hydrazyls mit langkettigen freien radikalen, die beim ultraschallabbau von polymethacrylsäuremethylester gebildet werden. Die Makromolekulare Chemie, 15, 188-210. doi:10.1002/macp.1955.020150115

[20] Price, G. (1990) The use of ultrasound for the controlled degradation of polymer solutions. In: Mason, T.J., Ed., Advances in Sonochemistry, JAI Press, London, 231-287.

[21] von Sonntag, C., Mark, G., Tauber, A. and Schuchmann, H.-P. (1999) OH radical formation and dosimetry in the sonolysis of aqueous solutions. Advances in Sonochemistry, 5, 109-145. doi:10.1016/S1569-2868(99)80004-7

[22] Lawal, O.S. (2009) Starch hydroxyalkylation: Physicochemical properties and enzymatic digestibility of native and hydroxypropylated finger millet (Eleusine coracana) starch. Food Hydrocolloids, 23, 415-425. doi:10.1016/j.foodhyd.2008.02.013

[23] Lorimer, J.P. (1990) Ultrasound in polymer chemistry. In: Mason, T.J., Ed., Sonochemistry: The use of ultrasound in chemistry, The Royal Society of Chemistry, Cambridge, 9-26,112-131.

[24] Price, G.J. (1994) Ultrasonically enhanced polymerization reactions. Trends in Polymer Science, 2, 174-177.

[25] Basedow, A.M. and Ebert, K.H. (1977) Ultrasonic degradation of polymers in solution. In: Advances Polymer 
Science, Springer-Verlag, New York, 22,88-148.

[26] Sohma, J. (1979) Electron spin resonance studies of the mechanical degradation of polymers. In: Grassie, N., Ed., Developments in Polymer Degradation, Elsevier, London, 99-127.

[27] Price, G.J. and Lenz, E.J. (1993) The use of dosimeters to measure radical production in aqueous sonochemical systems. Ultrasonics, 31, 451-456. doi:10.1016/0041-624X(93)90055-5

[28] Kanwal, F., Liggat, J.J. and Pethrick, R.A. (2000) Ultrasonic degradtion of polystyrene solutions. Polymer Degradation and Stability, $\mathbf{6 8}, 445-449$. doi:10.1016/S0141-3910(00)00034-3

[29] Tayal, A. and Khan, S.A. (2000) Degradation of a watersoluble polymer: Molecular weight changes and chain scission characteristics. Macromolecules, 33, 9488-9493. doi: $10.1021 / \mathrm{ma} 000736 \mathrm{~g}$

[30] Zhou, C. and Ma, H. (2006) Ultrasonic degradation of polysaccharide from a red algae (Porphyra yezoensis). Journal of Agricultural and Food Chemistry, 54, 22232228. doi:10.1021/if052763h

[31] Czechowska-Biskup, R., Rokita, B., Lotfy, S., Ulanski, P. and Rosiak, J.M. (2005) Degradation of chitosan and starch by $360-\mathrm{kHz}$ ultrasound. Carbohydrate Polymers, 60, 175-184. doi:10.1016/j.carbpol.2004.12.001

[32] Suslick, K. and Doktycz, S. (1990) Sounding out new chemistry. New Scientist, 3, 50-53.

[33] Suslick, K.S. (1988) Ultrasound, its chemical, physical and biological effects. VCH, Berlin.

[34] Suslick, K.S., Dokytyez, S. J. and Flint, E.B. (1990) On the origin of sonoluminescence and sonochemistry. Ultrasonics, 5, 280-290. doi:10.1016/0041-624X(90)90033-K

[35] Mason, T.J. (1990) Advances in Sonochemistry. Vol. 1, JAI Press, London, 231-287.

[36] Fisher, C.H., Hart, E.J. and Henglein, A. (1986) Ultrasonic irradiation of water. Journal of Phyical Chemistry, 90, 1954-1956.

[37] Henglein, A. and Kormann, C. (1985) Scavenging of $\mathrm{OH}$ radicals produced in the sonolysis of water. International Journal of Radiation in Biology, 48, 251-258. doi:10.1080/09553008514551241

[38] Lorimer, J.P. and Mason, T.J. (1995) Some recent studies at Coventry university, sonochemistry centre. Ultrasonics Sonochemistry, 2, S79-S86. doi:10.1016/1350-4177(95)00026-3

[39] Manousaki, E., Psillakis, E., Kalogerakis, N. and Mantzavinos, D. (2004) Degradation of sodium dodecylbenzene sulfonate in water by ultrasonic irradiation. Water Research, 38, 3751-3759. doi:10.1016/j.watres.2004.06.002

[40] Astrid, R., Michael, T. and Georg, G. (2004) Application of power ultrasound for azo degradation. Ultrasonics Sonochemistry, 11, 177-182. doi:10.1016/j.ultsonch.2004.01.030

[41] Portenlanger, G. and Heusinger, H. (1997) The influence of frequency on the mechanical and radical effects for the ultrasonic degradation of dextranes. Ultrasonics Sonochemistry, 4, 127-130.

doi:10.1016/S1350-4177(97)00018-7

[42] Trzcinski, S., Danuta U. and Staszewska, D.U. (2004) Kinetics of ultrasonic degradation and polymerisation degree distribution of sonochemically degraded chitosans. Carbohydrate Polymers, 56, 489-498. doi:10.1016/j.carbpol.2004.03.017

[43] Wasikiewicz, J.M., Yoshii, F., Nagasawa, N., Wach, R.A. and Mitomo, H. (2005) Degradation of chitosan and sodium alginate by gamma radiation, sonochemical and ultraviolet methods. Radiation Physics and Chemistry, 73, 287-295. doi:10.1016/j.radphyschem.2004.09.021

[44] Price, G.J., Smith, P.F. and West, P.J. (1991) Ultraonically initiated polymerization of methyl methacrylate. Ultrasonics, 29, 166-170. doi:10.1016/0041-624X(91)90047-C

[45] Chen, R.H., Chang, J.R. and Shyr, J.S. (1997) Effect of ultrasonic conditions and storage in acidic solutions on changes in molecular weight and polydispersity of treated chitosan. Carbohydrate Research, 299, 287-294. doi:10.1016/S0008-6215(97)00019-0

[46] Seguchi, M., Higasa, T. and Mori, T. (1994) Study of wheat starch structures by sonication treatment. Cereal Chemistry, 71, 636-639.

[47] Isono, Y., Kumagai, T. and Watanabe, T. (1994) Ultrasonic degradation of waxy rice starch. Bioscience Biotechnolgy and Biochemistry, 58, 1799-1802. doi:10.1271/bbb.58.1799

[48] Kasaai, M.R., Arul, J. and Charlet, G. (2008) Fragmentation of chitosan by ultrasonic irradiation. Ultrasonics Sonochemistry, 15, 1000-1008. doi:10.1016/j.ultsonch.2008.04.005

[49] Malhotra, S.L. (1982) Ultrasonic degradation of hydroxypropyl cellulose solutions in water, ethanol, and tetrahydrofuran. Journal of Macromolecule Science-Chemistry, A17, 601-636. doi:10.1080/00222338208062411

[50] Szorek, R. (1979) Ultrasonic degradation of polycaproamide in $40 \%$ sulfuric acid solution. Journal of Polymer Science, Polymer Physics, 17, 939-944. doi:10.1002/pol.1979.180170603

[51] Kruus, P., Lawrie, J.A.G. and O’Neill, M.L. (1988) Polymerization and depolymerization by Ultrasound. Ultrasonics, 26, 352-356. doi:10.1016/0041-624X(88)90035-2

[52] Keqiang, C., Ye, S., Huilin, L. and Xi, X.U. (1985) Studies on ultraonic degradation and block copolymerization of hydroxyethyl cellulose and poly (ethylene oxide). Journal of Macromolecule Science-Chemistry, A22, 455469.

[53] Sesshadri, R., Weiss, J., Hulbert, G.J. and Mount, J. (2003) Ultrasonic processing influences rheological and optical properties of high methoxyl pectin dispersions. Food Hydrocolloids, 17, 191-197. doi:10.1016/S0268-005X(02)00051-6

[54] Kawabata, K. and Umemura, S. (1993) Highly efficient sonochemical reaction with a switched spiral focal field. Ultrasonics, 31, 457-462. doi:10.1016/0041-624X(93)90056-6

[55] Kusters, K.A., Pratsinis, S., Thoma, S.G. and Smith, D.M. 
(1993) Ultrsonic fragmentation of agglomerate powders. Chemical Engineering Science, 48, 4119-4127. doi:10.1016/0009-2509(93)80258-R

[56] Ciftcioglu, M., Akinc, M. and Burkhart, L. (1986) Measurement of agglomerate strength distributions in agglomerated powders. Ceramic Bulletin, 65, 1591-1596.

[57] Friedman, V.M. (1972) The interaction mechanism between cavitation bubbles and particles of the solid and liquid phases. Ultrasonics, 19, 162-172. doi:10.1016/0041-624X(72)90357-5

[58] Nyborg, W.L. (1991) Biological effects of sound and ultrasound. In: Trigg, G.L., Ed., Encyclopedia of Applied Physics, Vol. 2, VCH, Berlin, 403-421.

[59] Qian, Z., Stoodely, P. and Pitt, W.G. (1996) Effect of lowintensity ultrasound upon biofilm structure from confocal scanning laser microscopy observation. Biomaterials, 17, 1975-1980. doi:10.1016/0142-9612(96)00022-1

[60] Mason, T.J. (1986) Use of ultrasound in chemical synthesis. Ultrasonics, 24, 245-253. doi:10.1016/0041-624X(86)90101-0

[61] Ley, S.V. and Low, C.M.R. (1989) Ultrasound in synthesis. Springer-Verlag, Berlin. doi:10.1007/978-3-642-74672-7

[62] Li, Y., Li, J., Guo, S. and Li, H. (2005) Mechanochemical degradation kinetics of high-density polyethylene melt and its mechanism in the presence of ultrasonic irradiation. Ultrasonics Sonochemistry, 12, 183-189. doi:10.1016/j.ultsonch.2003.10.011

[63] Mason, T.J. (1992) Industrial sonochemistry: Potential and practicality. Ultrasonics, 30, 192-196. doi:10.1016/0041-624X(92)90072-T

[64] Mason, T.J. (1997) Ultrasound in synthetic organic chemistry. Chemical Society Reviews, 26, 443-451. doi:10.1039/cs9972600443

[65] Price, G.J., White, A.J. and Clifton, A.A. (1995) The effect of high intensity ultrasound on solid polymers. Polymer, 26, 4919-4925. doi:10.1016/0032-3861(96)81616-8

[66] Roberts, R.T. (1993) High intensity ultrasonics in food processing. Chemistry and Industry, 15, 119-121.

[67] Cains, P.W., Martin, P.D. and Price, C.J. (1998) The use of ultrasound in industrial chemical synthesis and crystallization. 1. Applications to synthetic chemistry. Organic Process Research and Development, 1, 234-248. 